Vol. 38, No.5, pp. 182-193, Nov./Dec.2021 Revista Boliviana de Química

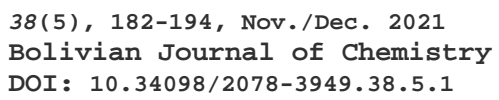

\title{
CARACTERIZACIÓN MECÁNICA Y MICROESTRUCTURAL DE UN ACERO INOXIDABLE AISI:304 ENDURECIDO MEDIANTE NITRURACIÓN USANDO RESIDUOS DE MELAMINA
}

\author{
Full original article
}

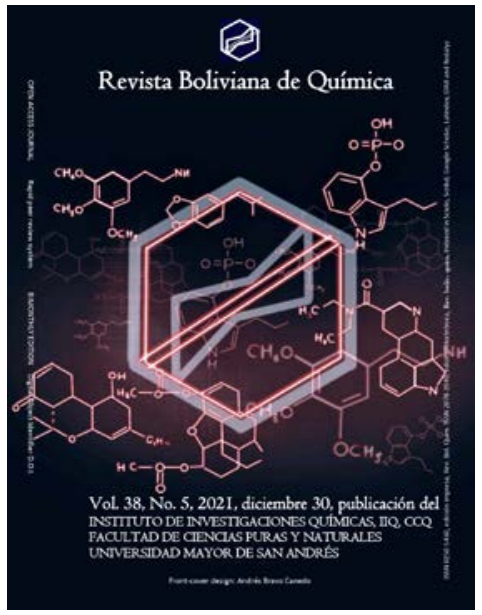

\section{Marcelo Rodríguez Valdivia*, Edgard Mollehuanca Caballero ${ }^{1}$}

Escuela Profesional de Ingeniería de Materiales, Facultad de Ingeniería de Procesos FIP, Universidad Nacional San Agustín de Arequipa UNSA, Av. Independencia s/n-Pab. Ing. Materiales, phone +5154200037, Arequipa, Perú, materiales@unsa.edu.pe, http://fip.unsa.edu.pe/ingmateriales/

Keywords: Melamine, diffusion, nitriding, factorial experimental design, stainless steel AISI:304, surface hardness

Palabras clave: Melamina, difusión, nitruración, diseño experimental factorial, acero inoxidable AISI:304, dureza superficial

\section{ABSTRACT}

This article presents the results obtained from the nitriding of an AISI: 304 stainless steel, subjected to a solid nitriding process recycling melanin residues; due to its chemical composition it constitutes a suitable material for supplying nitrogen gases. The micrographs showed that the nitriding process causes important structural changes with the formation of Fe nitrides $\left(\gamma^{\prime}-\mathrm{Fe}_{4} \mathrm{~N}\right.$ and $\left.\varepsilon-\mathrm{Fe}_{2} \mathrm{~N}\right)$, which substantially increase the surface hardness. Tests at $550^{\circ} \mathrm{C} / 4$ hours, $580^{\circ} \mathrm{C} / 4$ hours and $565^{\circ} \mathrm{C} / 3$ hours, showed surface hardness of 30.17, 30.0 and 29.9 HRc; representing an increase of $67 \%$ when compared to steel in supply condition. Statistical analysis applying a factorial experimental design indicated that the main factors that influence hardness are: nitriding time and its interaction with temperature with a significance of 0.0094 and 0.0707 for a confidence level of $90 \%$. The thickness of the hardened layer is directly dependent on the concentration and diffusion rate of $\mathrm{N}$ atoms that enter the steel from its surface and move towards the interior.

*Correspondent autor: mrodriquezv@unsa.edu.pe

\section{RESUMEN}

Downloadable from: Revista Boliviana de Química http://www.bolivianchemistryjournal.org
182

Volumen 38 No5. Año 2021

http://www.scribd.com/bolivianjournalofchemistry 
Este artículo presenta los resultados obtenidos en la nitruración de un acero inoxidable AISI: 304, sometido a un proceso de nitruración sólida reciclando residuos de melanina; que por su composición química constituye un adecuado material para el aporte de gases de nitrógeno. Las micrografías mostraron que el proceso de nitruración causa importantes cambios estructurales con formación de nitruros de $\mathrm{Fe}\left(\gamma^{\prime}-\mathrm{Fe}_{4} \mathrm{~N}\right.$ y $\left.\varepsilon-\mathrm{Fe}_{2} \mathrm{~N}\right)$, que incrementan sustancialmente la dureza superficial. Pruebas a $550^{\circ} \mathrm{C} / 4$ horas, $580^{\circ} \mathrm{C} / 4$ horas y $565^{\circ} \mathrm{C} / 3$ horas, presentaron durezas superficiales de 30.17, 30.0 y 29.9 HRc; que representan un incremento del 67\% cuando se compara con el acero en estado de suministro. El análisis estadístico aplicando un diseño experimental factorial, indicó que los principales factores que influyen sobre la dureza son: el tiempo de nitruración y su interacción con la temperatura con una significancia de 0.0094 y 0.0707 para un nivel de confianza de $90 \%$.

El espesor de la capa endurecida, es dependiente directamente de la concentración y de la velocidad de difusión de átomos de $\mathrm{N}$ que ingresan en el acero desde su superficie y se movilizan hacia el interior.

\section{INTRODUCCIÓN}

El acero inoxidable AISI: 304 se utiliza en aplicaciones donde deben tener buena resistencia al desgaste y a la corrosión. Para lograr esto, se les aplica un tratamiento termoquímico denominado nitruración para aumentar su dureza superficial.

La nitruración consiste en el endurecimiento de la superficie de aceros al carbono y aleados en una atmósfera que contiene una mezcla de gases de amoniaco y amoniaco disociado. La nitruración se suele realizar a una temperatura entre 500 y $600^{\circ} \mathrm{C}$ (nitruración a baja temperatura), por lo que no se produce ninguna transformación de fase [1]. Este proceso térmico, depende de la formación de nitruros en el acero por la reacción del nitrógeno con Fe y otros elementos de aleación. Aunque todos los aceros son capaces de formar nitruros de hierro a temperaturas y atmósferas adecuadas, los mejores resultados se obtienen con aceros que contienen uno o más de los principales elementos de aleación que forman nitruros. Estos elementos son aluminio, cromo, vanadio y molibdeno. La nitruración le confiere al acero una alta dureza de la capa superficial, que no cambia cuando se calienta hasta 400 o $450^{\circ} \mathrm{C}$, una elevada resistencia al desgaste [2, 3] y alta capacidad para soportar esfuerzos de fatiga.

Si se observa la capa del borde de las partes nitruradas bajo el microscopio óptico, se puede ver que consta de dos capas de aspecto diferente: una capa exterior blanca (capa compuesta), muy delgada y muy frágil, y una capa interior oscura más gruesa (zona de difusión), que es realmente importante. Se ha demostrado que esta última capa está formada por nitruros de elementos aleados y, por otro lado, en la exterior blanca (capa compuesta), además de los nitruros aleados, también se encuentra el nitruro de hierro. Por encima de $400^{\circ} \mathrm{C}$, el amoniaco se descompone según la reacción $\mathrm{NH}_{3} \rightarrow 3 \mathrm{H}+\mathrm{N}$. El nitrógeno atómico se difunde en el acero y forma fases de nitruro. A la temperatura de $591^{\circ} \mathrm{C}$, la capa de nitruro consta de tres fases: nitruro de $\varepsilon-\mathrm{Fe}_{2} \mathrm{~N}$, nitruro de $\mathrm{Y}^{\prime}-\mathrm{Fe}_{4} \mathrm{~N}$, que contiene $0.01 \%$ de nitrógeno a temperatura ambiente [4].

Estas capas duras se crean cuando el nitrógeno atómico se pone en contacto con la superficie del acero a la temperatura de nitruración $\left(500^{\circ} \mathrm{C}\right)$. Cuando el nitrógeno se difunde desde la superficie hacia el interior del acero [5], forma nitruros en la zona del borde, lo que causa un aumento de la dureza.

El nitrógeno utilizado en este proceso proviene del amoniaco, que al entrar en contacto con el acero a alta temperatura, que actúa como un agente catalizador, se disocia en nitrógeno e hidrógeno atómico según las reacciones (1), (2) y (3):

$$
2 \mathrm{NH}_{3} \longrightarrow 2 \mathrm{~N}+3 \mathrm{H}_{2}
$$

El nitrógeno que no se adsorbe pasa a molecular

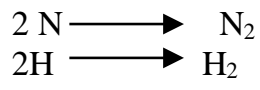

La melamina (2,4,6-triamino-1,3,5-triazina) es un compuesto orgánico con fórmula química $\mathrm{C}_{3} \mathrm{H}_{6} \mathrm{~N}_{6}$ y con un contenido de nitrógeno de 66\% [6]. A menudo se combina con formaldehído para ser utilizado en la fabricación de plásticos, incluidas pizarras blancas, utensilios de cocina y comerciales filtros. Las resinas de melamina se conocen como plástico termoestable, porque el plástico se fija después del moldeado. Si se expone a suficiente calor, las resinas de melamina se derriten. Sin embargo, el plástico es capaz de soportar temperaturas más altas que otros plásticos haciendo difícil su reciclaje. Debido a que la melanina es un material polimérico, tiene sus problemas

Downloadable from: Revista Boliviana de Química http://www.bolivianchemistryjournal.org
183

Volumen 38 N5. Año 2021 http://www.scribd.com/bolivianjournalofchemistry 
<smiles>Nc1nc(N)nc(N)n1</smiles>

Figura 1. Fórmula estructural de melamina

El presente estudio se ha dividido en dos etapas. En la primera, se ha realizado la preparación del material nitrurante, el mecanizado de las muestras de acero y el tratamiento termoquímico de nitruración. En la segunda etapa, se realizaron los ensayos de dureza superficial y la caracterización por microscopía óptica para identificar las microestructuras nitruradas. Adicionalmente, se determinaron los espesores de capa nitrurada mediante la aplicación de software de análisis de imágenes.

\section{EXPERIMENTAL}

\section{Materiales y métodos}

\section{Materiales}

Está compuesto por muestras de acero inoxidable AISI: 304, adquirido del proveedor Aceros del Perú S.A.C; que han sido mecanizados con dimensiones según la norma técnica ASTM E-23 [7]. El material que suministra nitrógeno gaseoso consiste en residuos de melanina (vajilla comercial inservible) provenientes de la actividad gastronómica.

\section{Reactivos}

Se emplearon los siguientes reactivos químicos: $\mathrm{CuSO}_{4} .5 \mathrm{H}_{2} \mathrm{O}$ (99.99\%), $\mathrm{HCl}$ (37\%), alcohol etílico (97.0\%), adquiridos de la empresa Diproquim.

\section{Metodología}

\section{Preparación del material Nitrurante (Melamina)}

A fin de conseguir fragmentos de melanina entre 4 y $12 \mathrm{~mm}$. de longitud, se procedió a realizar la fragmentación empleando un mortero de hierro fundido y luego el tamizado necesario para obtener un material homogéneo. Se utilizó 120 g. de melanina fragmentada por cada prueba de nitruración. En las Figuras 2(a) y 2(b), se muestran los materiales utilizados para la realización de las pruebas de nitruración.

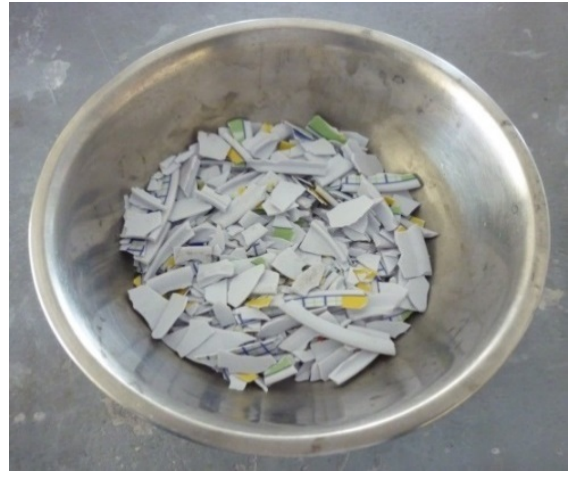

(a)

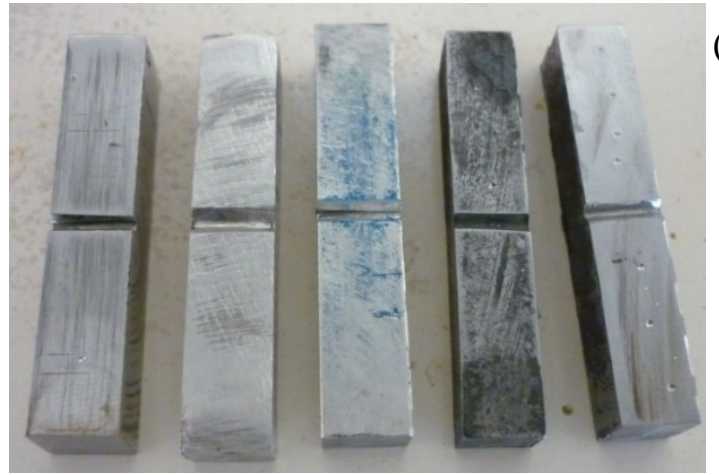

(b)

Figura 2. Materiales: (a) Fragmentos de melanina, (b) Muestras de acero AISI: 304

Downloadable from: Revista Boliviana de Química http://www.bolivianchemistryjournal.org
184

Volumen 38 N5. Año 2021 http://www.scribd.com/bolivianjournalofchemistry 


\section{Tratamiento Termoquímico de Nitruración}

Las pruebas experimentales de nitruración se llevaron a cabo empleando recipientes cilíndricos de diferentes tamaños, donde se colocaron muestras de acero inoxidable AISI 304 previamente lavadas y secadas con aire caliente. Una cantidad de $120 \mathrm{~g}$ de melanina fragmentada se colocó en el fondo del recipiente hasta alcanzar una altura de $2 \mathrm{~cm}$. Seguidamente se colocaron las muestras de acero cubriéndolas totalmente con la melanina restante. A fin de evitar la fuga de gases de nitrurantes, los contenedores fueron sellados con una tapa cerámica hecha de una mezcla de arcilla refractaria, arena silícea y silicato de sodio. De acuerdo al diagrama de la Figura 3, el proceso termoquímico se debe llevar a cabo empleando temperaturas de 550,565 y $580^{\circ} \mathrm{C}$.

El tiempo de permanencia en el interior del horno se determinó en 2, 3 y 4 horas respectivamente. Una vez terminado el tratamiento, las muestras se dejaron enfriar a temperatura ambiente. Procedimiento experimental similar al reportado en [8]. La Figura 3, presenta el diagrama del proceso del tratamiento termoquímico.

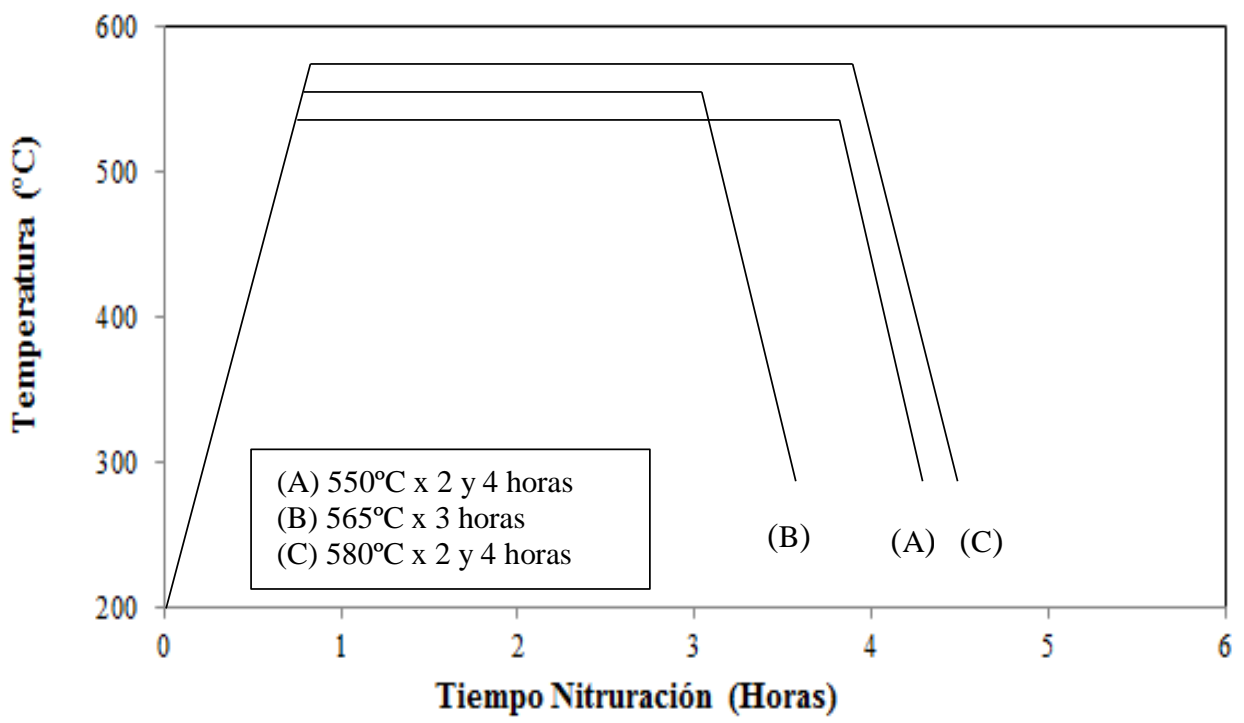

Figura 3. Diagrama del tratamiento termoquímico

Para el calentamiento de las muestras se utilizó un horno de resistencias eléctricas marca Veb Elektro Bad Frankenhausen con controlador digital de temperatura de 0 a $1200^{\circ} \mathrm{C}$. La Figura 4, muestra el recipiente metálico en el interior del horno de resistencias eléctricas.

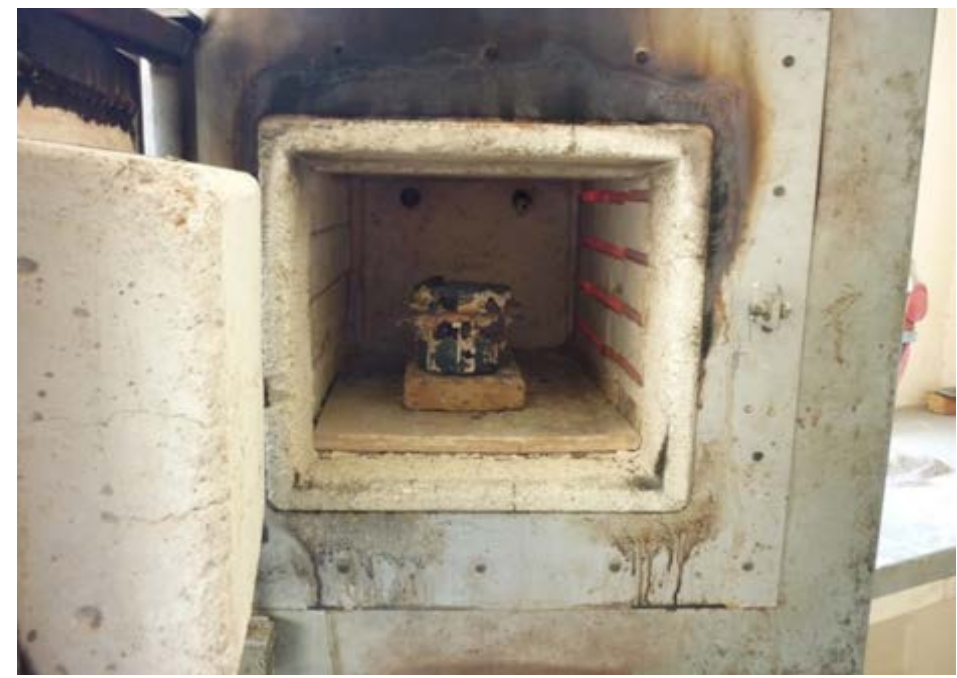

Figura 4. Horno para el tratamiento termoquímico de nitruración

Downloadable from: Revista Boliviana de Química http://www.bolivianchemistryjournal.org
185

Volumen 38 N5. Año 2021 http://www.scribd.com/bolivianjournalofchemistry 


\section{Equipos de ensayos y caracterización}

La medición de la dureza superficial se determinó utilizando un durómetro digital INDENTEC, empleando la escala Rockwell C con aplicación de una carga de 150 kg-f y un micro indentador de cono de diamante. La ejecución del ensayo fue siguiendo lo indicado en la norma ASTM E-18 [9]. La observación microscópica de las fases microestructurales nitruradas se realizó mediante el uso de un microscopio metalográfico METALAB de luz reflejada, provisto de un conjunto de lentes objetivos de 5, 10, 20 y 50x [10]. Para la preparación de muestras metalográficas, se siguió el siguiente procedimiento propuesto por la norma ASTM E-3 [11].

1. Montaje de muestras en resina acrílica

2. Desbaste de muestras utilizando lijas al agua de grano \#220, 320, 400, 600

3. Pulido de muestras solución conteniendo alúmina de $1.0 \mu \mathrm{m}$.

4. Ataque químico para revelar las fases micro estructurales con reactivo de Marble’s (10 g. de $\mathrm{CuSO}_{4}$ en $50 \mathrm{~mL}$ de $\mathrm{H}_{2} \mathrm{O}$ y $50 \mathrm{~mL}$ de $\mathrm{HCl}$ ).

La toma de macrografías de las fracturas se realizó empleando un microscopio estereográfico Carl Zeiss con lentes objetivos de 0.5 a $5 x$. Para tal efecto, las superficies fracturadas fueron limpiadas con aire forzado para asegurar que estén libres de partículas extrañas.

\section{Medición del espesor de capa nitrurada}

Se realizó midiendo el espesor de la zona de fractura próxima a la superficie y que presenta grano fino (de apariencia brillante). Se utilizó el software de análisis de imágenes Image 4 Plus. De acuerdo con Bobadilla, M. [12], mediante la ecuación (1), es posible determinar la velocidad de difusión de N para las temperaturas de prueba y su correlación con el espesor de capa nitrurada [13].

$$
D=\operatorname{Doxp}\left(\frac{-Q}{R * T}\right)
$$

Donde: D es la difusividad ( $\left.\mathrm{cm}^{2} / \mathrm{s}\right)$, Do es constante de proporcionalidad ( $\left.\mathrm{cm}^{2} / \mathrm{s}\right)$, Q es la energía de activación de las especies en difusión (cal/mol), R es constante molar de los gases $\left(8.314 \mathrm{~J} / \mathrm{mol}{ }^{\circ} \mathrm{K}\right)$, T es la temperatura $\left({ }^{\circ} \mathrm{K}\right)$.

\section{Diseño experimental factorial a dos niveles}

Con el fin de evaluar el efecto de las variables experimentales sobre el proceso de la dureza superficial, se utilizó un diseño experimental factorial a dos niveles. Este diseño es muy consistente y permite definir las condiciones óptimas del proceso mediante el análisis de la varianza. Para tal propósito se empleó el software STATISTIC V.5. Se evaluaron dos factores independientes: temperatura y tiempo de nitruración. La respuesta dependiente fue la dureza superficial. En la Tabla 1, se describen los factores y niveles evaluados.

Tabla 1. Factores y niveles del diseño factorial

\begin{tabular}{lcc}
\hline Factores & Nivel (-) & Nivel (+) \\
\hline Temperatura de nitruración $\left({ }^{\circ} \mathrm{C}\right)$ & 550 & 580 \\
Tiempo de nitruración (horas) & 2 & 4 \\
\hline
\end{tabular}

Se realizaron 5 pruebas (réplicas) a la condición de $565^{\circ} \mathrm{C}$ y 3 horas para aumentar la precisión del modelo matemático.

\section{RESULTADOS Y DISCUSIONES}

Caracterización del acero AISI: 304

\section{Composición química}

Downloadable from: Revista Boliviana de Química http://www.bolivianchemistryjournal.org
186

Volumen 38 N5. Año 2021

http://www.scribd.com/bolivianjournalofchemistry 
Marcelo Rodríguez Valdivia et Edgard Mollehuanca Caballero RBQ Vol.38, No.5, pp. 182-193, 2021

La Tabla 2, presenta la composición química del acero y que corresponde a aquella indicada por el catálogo de productos del proveedor.

Tabla 2. Composición Química - acero inoxidable AISI: 304

\begin{tabular}{llll}
\hline Elemento & $\mathbf{C}$ & $\mathbf{C r}$ & $\mathbf{N i}$ \\
\hline Medida & $\%$ & $\%$ & $\%$ \\
& 0.07 & $17.0-19.5$ & $8.0-10.5$
\end{tabular}

\section{Microestructura del acero AISI: 304 sin tratamiento}

La Figura 5, muestra una microestructura irregular con granos austeníticos y partículas de carburos dispersos.

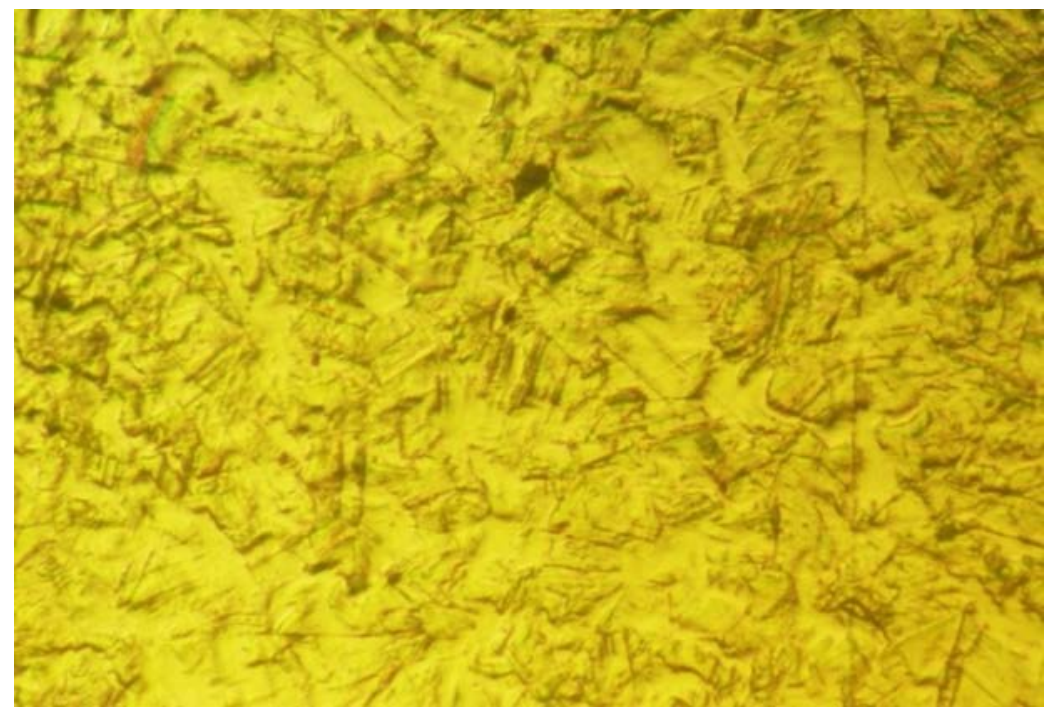

Figura 5. Micrografía al microscopio óptico del acero AISI: 304. Aumento 500x

\section{Caracterización de la melanina}

\section{Composición química}

La referencia bibliográfica revisada [14], da a conocer que la melamina contiene contenidos de carbono, hidrogeno y nitrógeno. La Tabla 3, presenta el análisis pirolítico y las características térmicas de la melamina.

Tabla 3. Análisis químico y propiedades térmicas [14]

\begin{tabular}{lccccc}
\hline & \multicolumn{3}{c}{ Composición (\%) } & Punto de Descomposición $\left({ }^{\circ} \mathbf{C}\right)$ & Peso Molecular \\
\cline { 2 - 4 } & $\mathbf{C}$ & $\mathbf{H}$ & $\mathbf{N}$ & 259 & Bajo \\
\hline Melamina en polvo & 40.4 & 5.71 & 26.6 & 316 & Alto \\
Melamina fragmentada & 35.8 & 5.15 & 34.8 & & \\
\hline
\end{tabular}

\section{Evaluación de la dureza superficial}

La Tabla 4, muestra los valores de dureza superficial determinados en muestras a diferentes condiciones de prueba, y la presentada por la muestra sin tratamiento.

Downloadable from: Revista Boliviana de Química http://www.bolivianchemistryjournal.org
187

Volumen 38 N5. Año 2021

http://www.scribd.com/bolivianjournalofchemistry 
Tabla 4. Dureza superficial (HRc)

\begin{tabular}{ccc}
\hline Prueba & Condiciones de Prueba & Dureza (HRc) \\
\hline 1 & $550^{\circ} \mathrm{C} / 2$ horas & 27.80 \\
2 & $580^{\circ} \mathrm{C} / 2$ horas & 29.33 \\
3 & $550^{\circ} \mathrm{C} / 4$ horas & 30.17 \\
4 & $580^{\circ} \mathrm{C} / 4$ horas & 30.00 \\
5 & $565^{\circ} \mathrm{C} / 3$ horas & 29.93 \\
6 & $565^{\circ} \mathrm{C} / 3$ horas & 29.00 \\
7 & $565^{\circ} \mathrm{C} / 3$ horas & 29.90 \\
8 & $565^{\circ} \mathrm{C} / 3$ horas & 29.73 \\
9 & $565^{\circ} \mathrm{C} / 3$ horas & 29.10 \\
10 & Acero AISI: 304 sin nitruración & 10.00
\end{tabular}

\section{Efecto de la temperatura de nitruración}

La Figura 6, muestra el efecto de la temperatura de nitruración sobre la dureza superficial para pruebas con un tiempo de tratamiento de 2 y 4 horas.

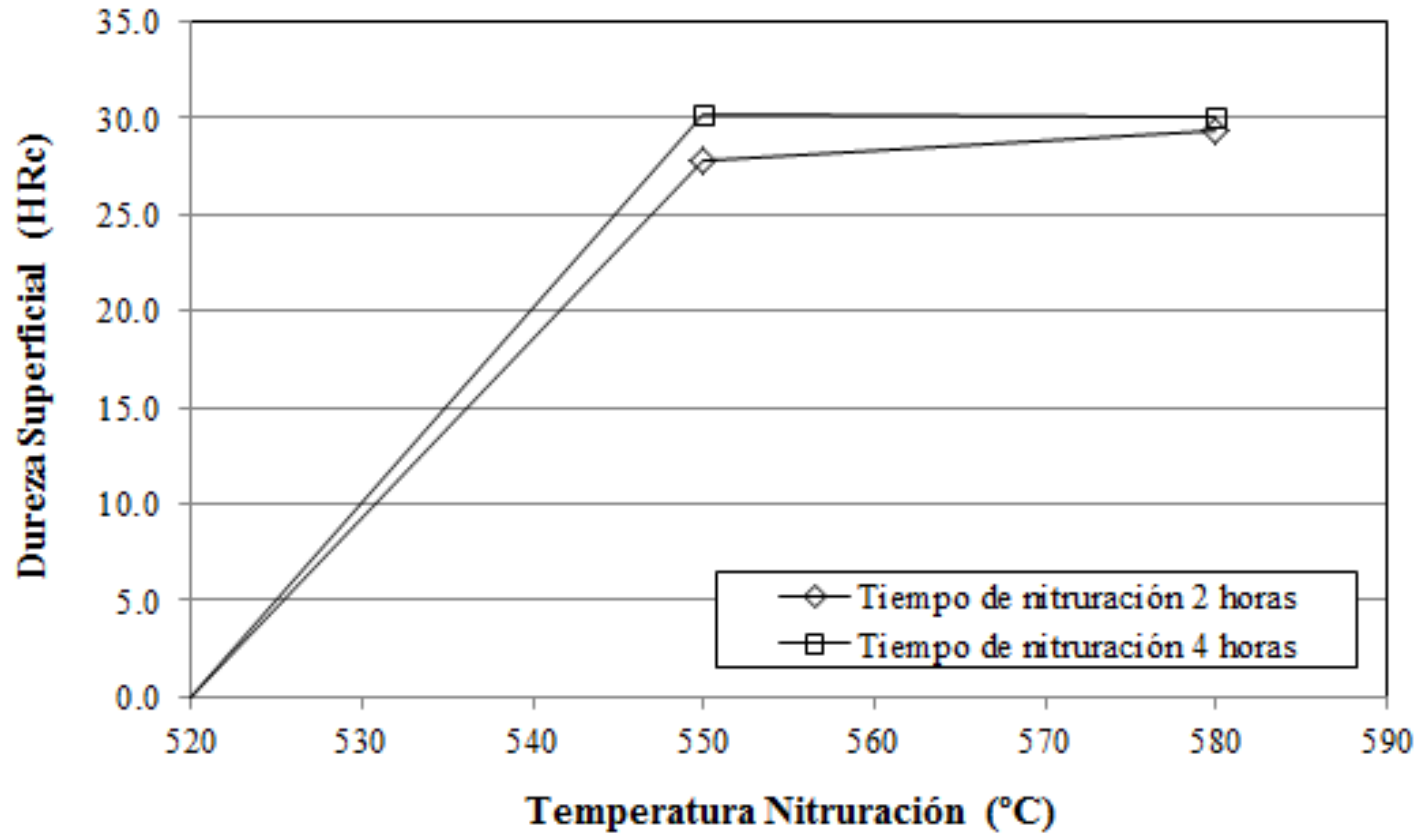

Figura 6. Dureza superficial en función de la temperatura de nitruración

\section{Efecto del tiempo de nitruración}

La Figura 7, muestra el efecto del tiempo de nitruración sobre la dureza superficial para pruebas a 550 y $580^{\circ} \mathrm{C}$.

Downloadable from: Revista Boliviana de Química http://www.bolivianchemistryjournal.org
188

Volumen 38 N5. Año 2021

http://www.scribd.com/bolivianjournalofchemistry 


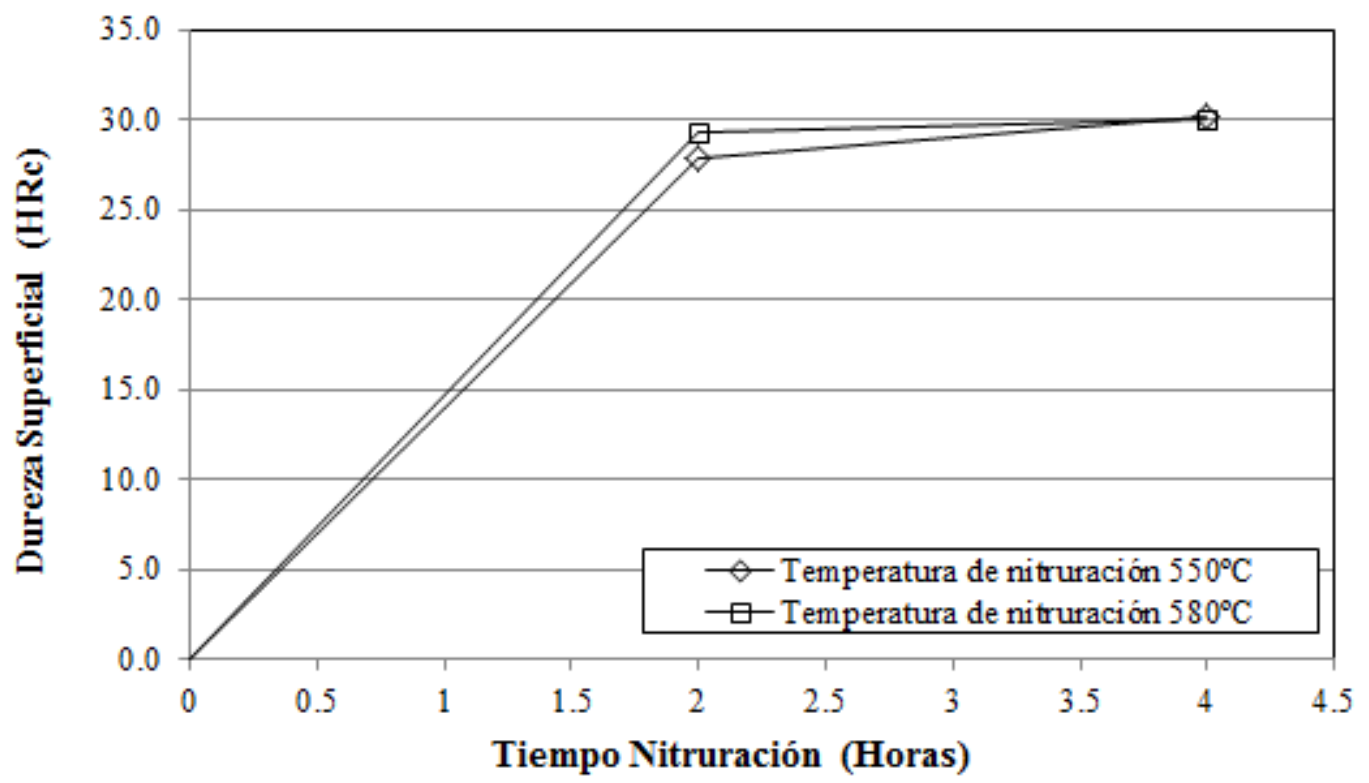

Figura 7. Dureza superficial en función del tiempo de nitruración

\section{Análisis estadístico ANOVA}

La Tabla 5, presenta los resultados del análisis de varianza (ANOVA) para el modelo factorial considerando el efecto de cada factor (1), (2); así como el efecto causado por sus interacciones 1*2. El resultado del análisis ANOVA para el modelo factorial considera la suma de los cuadrados de los tratamientos (SS), los grados de libertad (df), la suma de los cuadrados de los medios (MS).

La evaluación de la incidencia del tratamiento de los efectos se realizó mediante el uso de la distribución F. El estadístico valor-p, permite la comparación con el valor de significancia $\alpha=0.10$. Si $\mathrm{p}<\alpha$, se rechaza la hipótesis nula y se concluye que el correspondiente efecto está activo o influye en la respuesta. La cantidad R-sqr $=0.83525$, indica que se obtienen valores de dureza superficial con aproximadamente un $83.53 \%$ de variabilidad, mientras que Adj. (R-sqr adj.) igual a 0.73639; corresponde al valor ajustado al tamaño o al número de factores en el modelo.

Tabla 5. Análisis de varianza para el modelo factorial ANOVA; Var. DUREZA; R-sqr= 0.83525; Adj: $0.73639 ; 2 * *(2-0)$ desing: MS Residual =0.1378911 DV:

\begin{tabular}{lccccc}
\hline \multicolumn{1}{c}{ Factor } & SS & df & MS & Fo & p \\
\hline (1) Temperatura de nitruración & 0.462400 & 1 & 0.462400 & 3.35337 & 0.126569 \\
(2) Tiempo de nitruración & 2.310400 & 1 & 2.310400 & 16.75525 & 0.009416 \\
$1 * 2$ & 0.722500 & 1 & 0.722500 & 5.23964 & 0.070735 \\
Error & 0.689456 & 5 & 0.137891 & & \\
Total SS & 4.184756 & 8 & & & \\
\hline
\end{tabular}

Del análisis de Fo, se aprecia que el efecto más significativo es el tiempo de nitruración y la interacción entre la temperatura y el tiempo nitruración.

\section{Microestructuras Nitruradas}

La Figura 8, muestra las microestructuras obtenidas por microscopía óptica. Se puede observar la formación de capas nitruradas conformadas por una capa compuesta y una zona de difusión.

Downloadable from: Revista Boliviana de Química http://www.bolivianchemistryjournal.org
189

Volumen 38 N5. Año 2021

http://www.scribd.com/bolivianjournalofchemistry 

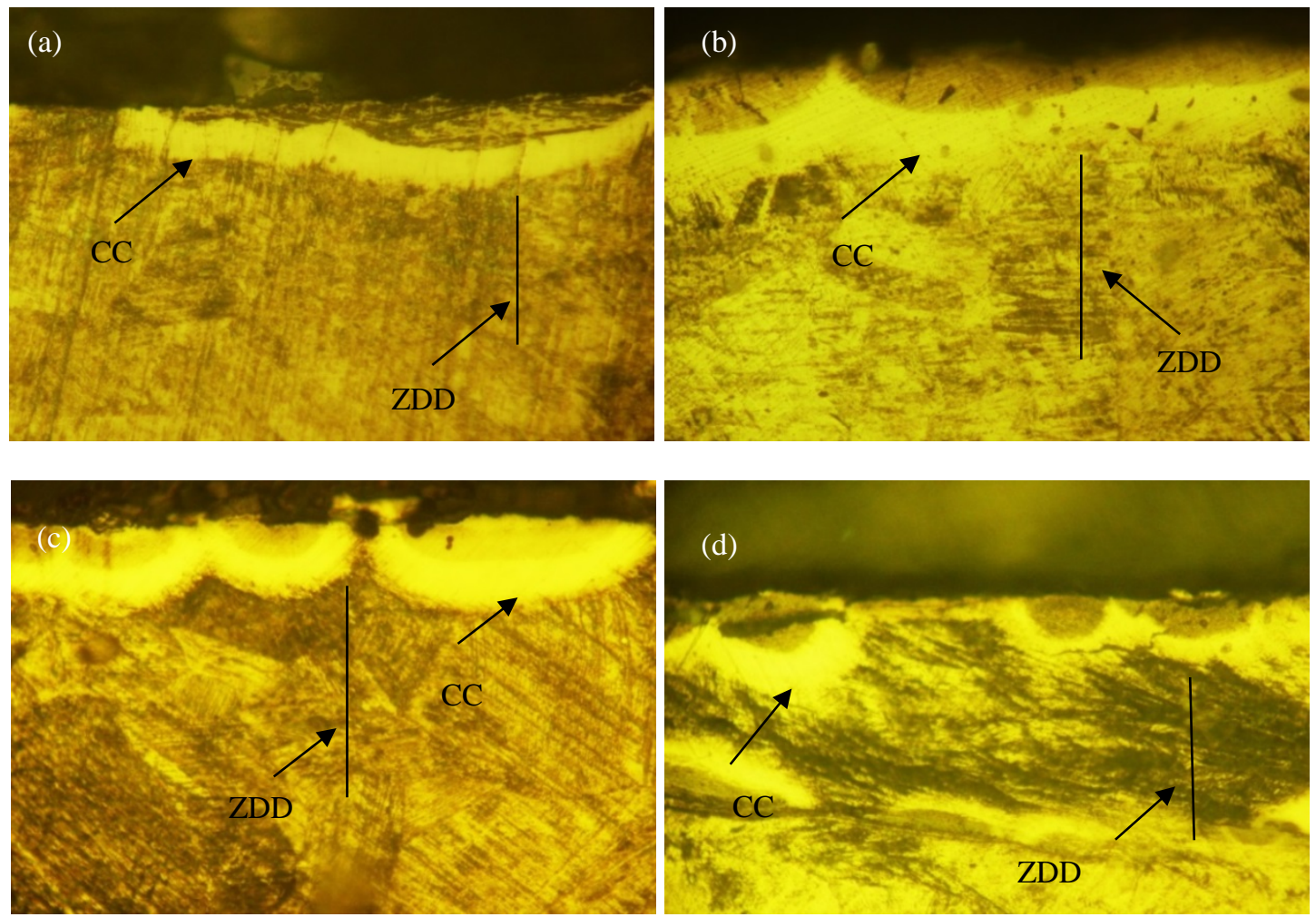

Figura 8. Microestructuras nitruradas mostrando Capa compuesta (CC) y Zona de Difusión (ZDD)

(a) Prueba a $550^{\circ} \mathrm{C} / 4$ horas, (b) Prueba a $580^{\circ} \mathrm{C} / 4$ horas, (c y d) Prueba a $565^{\circ} \mathrm{C} / 3$ horas. Aumento 500x.

En la zona de capa compuesta (CC) se forman principalmente nitruros del tipo $Y^{\prime}-\mathrm{Fe}_{4} \mathrm{~N}_{1-\mathrm{x}}$ y $\varepsilon-\mathrm{Fe}_{2} \mathrm{~N}_{1-\mathrm{z}}$. La observación al microscopio presenta un aspecto de capa blanca cuya principal característica es su elevada resistencia al desgaste y a la corrosión. En la zona de difusión (ZDD), el nitrógeno se difunde desde la superficie hacia el núcleo del material; su profundidad depende del tiempo y la temperatura. Benlahreche, F. Z. [15], identifica una compuesta y una zona de difusión en pruebas de nitruración de un acero da baja aleación. Parte del nitrógeno se precipita el nitruro $\gamma^{\prime}-\mathrm{Fe}_{4} \mathrm{~N}$ en forma de agujas. Las fases microestructurales obtenidas sugieren que cuando el nitrógeno difunde hacia el interior del acero, la fase Y’(Fe gama) se sobresatura y forma la capa compuesta [16, 17].

\section{Espesor de capa nitrurada}

En la Figura 9 y la Tabla 6, se presentan la medición del espesor de la capa nitrurada y los espesores promedio medidos usando el software de análisis de imágenes.

Tabla 6. Espesores promedios de capas nitruradas

\begin{tabular}{cccc}
\hline $\begin{array}{c}\text { Temperatura de nitruración } \\
\left({ }^{\circ} \mathrm{C}\right)\end{array}$ & $\begin{array}{l}\text { Tiempo de nitruración } \\
(\text { horas })\end{array}$ & $\begin{array}{c}\text { Espesor } \\
(\mathrm{mm})\end{array}$ & $\begin{array}{c}\text { Espesor } \\
(\mu \mathrm{m})\end{array}$ \\
\hline 550 & 4 & 0.029 & 29.0 \\
580 & 4 & 0.046 & 46.0 \\
565 & 3 & 0.046 & 46.0 \\
565 & 3 & 0.036 & 36.0 \\
565 & 3 & 0.043 & 43.0 \\
565 & 3 & 0.054 & 54.0 \\
565 & 3 & 0.045 & 45.0 \\
\hline
\end{tabular}

Downloadable from: Revista Boliviana de Química http://www.bolivianchemistryjournal.org 


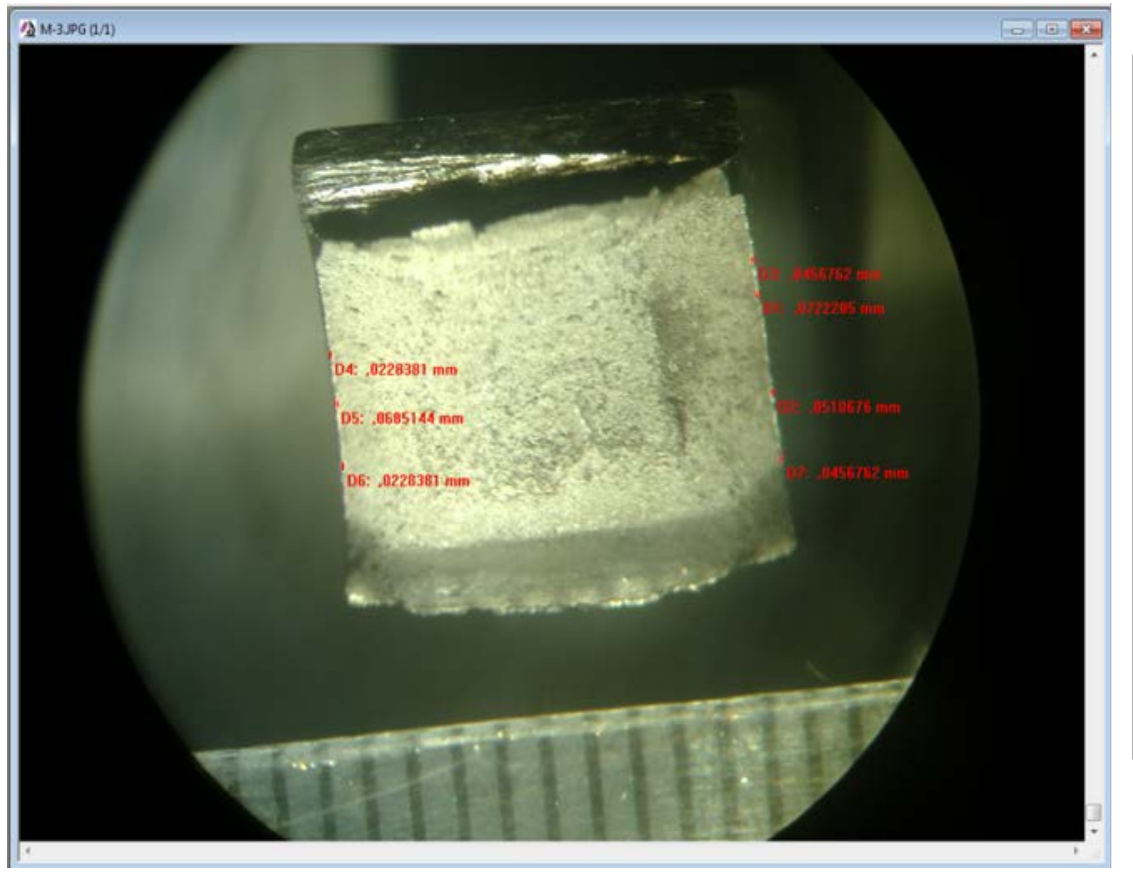

\begin{tabular}{lr} 
D1 &, $0722205 \mathrm{~mm}$ \\
D2 &, $0510676 \mathrm{~mm}$ \\
D3 &, $0456762 \mathrm{~mm}$ \\
D4 &, $0228381 \mathrm{~mm}$ \\
D5 &, $0685144 \mathrm{~mm}$ \\
D6 &, $0228381 \mathrm{~mm}$ \\
D7 &, $0456762 \mathrm{~mm}$ \\
Min. &, $0228381 \mathrm{~mm}$ \\
Max. &, $0722205 \mathrm{~mm}$ \\
Range &, $0493823 \mathrm{~mm}$ \\
Mean &, $0469759 \mathrm{~mm}$ \\
Std. Dev, $0180735 \mathrm{~mm}$ \\
$\begin{array}{lr}\text { Sum } & , 3288314 \mathrm{~mm} \\
\text { No. of Samples } 7\end{array}$ \\
\hline
\end{tabular}

Figura 9. Medición de espesor de capa nitrurada. Prueba $550^{\circ} \mathrm{C} / 4$ horas.

Los resultados obtenidos en las pruebas de nitruración del acero inoxidable AISI: 304, usando residuos de un polímero termoestable (melanina) evidenciaron que:

La nitruración se ha desarrollado mediante el nitrógeno atómico procedente de la descomposición de combinaciones nitrogenadas, como el amoníaco, que es generado cuando la melanina se quema. Se producen 3 etapas: 1) disociación térmica del amoníaco, 2) absorción del N por el Fe para formar primeramente solución sólida y después nitruro de hierro $\left(\mathrm{Fe}_{4} \mathrm{~N}\right)$ y 3 ) difusión del nitrógeno hacia el interior del acero. Ocurren las reacciones (4) y (5).

$$
\begin{array}{cl}
2 \mathrm{NH}_{3} & \longrightarrow 2 \mathrm{~N}+3 \mathrm{H}_{2} \quad(4) \\
\mathrm{N}+\mathrm{Fe} & \longrightarrow \mathrm{Fe}_{\mathrm{x}} \mathrm{N} \text { (pudiendo ser } \mathrm{Fe}_{4} \mathrm{~N}, \mathrm{Fe}_{3} \mathrm{~N} \text { o } \mathrm{Fe}_{2} \mathrm{~N} \text { ) }
\end{array}
$$

El N no absorbido inmediatamente pasa a molecular y ya no actúa como nitrurante, según la reacción (6).

$$
2 \mathrm{~N} \longrightarrow \mathrm{N}_{2}
$$

\section{Dureza superficial}

\section{Efecto de la temperatura y tiempo de nitruración}

La temperatura y el tiempo son los factores determinantes de las características de la capa nitrurada. El correcto control de éstas depende la dureza superficial, espesores de capa; dándose una cinética de difusión en su formación. $\mathrm{Al}$ incrementarse la temperatura y el tiempo se consigue variar las constantes de difusión del N, ello debido al tipo de nitruro que se forma tanto en la superficie, como en la zona sub-superficial nitrurada.

$\mathrm{Al}$ incrementar el tiempo la intensidad de la fase $\varepsilon$-Fe2-3N disminuye, mientras que la fase $\gamma^{\prime}$-Fe4N se incrementa. La fase $\gamma^{\prime}$-Fe4N es dura y frágil, el incremento de esta fase aumentará la dureza de la capa compuesta [18]. Además, el elemento Cr (17.0-19.5\%) presente en el acero inoxidable AISI: 304 forma CrN que favorece también al aumento de la dureza [19]. Lin, Y, et. al. [20], afirman que el incremento de la dureza con el tiempo se debe al aumento del espesor de capa nitrurada y al alto contenido de $\mathrm{N}$ en ésta.

\section{Diseño experimental factorial}

Las variables que influyen directamente en el proceso de nitruración son: el tiempo de nitruración, la interacción entre la temperatura y el tiempo de nitruración con una probabilidad del 90\%. El análisis de varianza de los resultados

Downloadable from: Revista Boliviana de Química http://www.bolivianchemistryjournal.org
191

Volumen 38 N5. Año 2021 http://www.scribd.com/bolivianjournalofchemistry 

más óptima. El siguiente modelo matemático expresa lo afirmado anteriormente, y puede ser validado manteniendo las mismas condiciones experimentales.

\section{Dureza Superficial $($ HRc $)$ = - 33.7494 + 0.1760 (Temperatura Nitruración) + 16.7683 (Tiempo Nitruración) - 0.0283 (Temperatura Nitruración)*(Tiempo de Nitruración)}

\section{Microestructuras nitruradas}

En las muestras que se realizaron nitruración a $550^{\circ} \mathrm{C}$ por 4 horas, $565^{\circ} \mathrm{C}$ por 3 horas y $580^{\circ} \mathrm{C}$ por 4 horas; se evidencian claramente la formación de nitruros de Fe que se encuentran presentes en la capa compuesta (capa blanca) y en la zona de difusión (estructura con forma de agujas). Ferreira, L.M. et. al. [21], destaca la formación de capas blancas luego de la nitruración de un acero AISI 420 a $450^{\circ} \mathrm{C}$ por 4 horas. La presencia de estos nitruros son los causantes del incremento de la dureza superficial [22]. Es posible afirmar que bajo estas condiciones de prueba se ha mantenido una alta concentración de $\mathrm{N}$ muy cercana al $20 \%$.

\section{Espesor de capa nitrurada}

El espesor de la capa nitrurada es determinado por la velocidad de difusión de nitrógeno en la superficie de acero. Por otro lado, su desarrollo se aproxima a una dependencia parabólica del tiempo para las primeras horas de nitruración hasta que se alcanza un grosor límite, luego del cual no se obtiene ningún crecimiento adicional. En pruebas a $550^{\circ} \mathrm{C}$ por 4 horas, $565^{\circ} \mathrm{C}$ por 3 horas y $580^{\circ} \mathrm{C}$ por 4 horas; se presentan espesores promedio entre $36.0 \mathrm{y}$ $54.0 \mu \mathrm{m}$., lo que indica que un flujo alto de átomos de nitrógeno ha difundido desde la superficie hacia el interior del acero. Los espesores obtenidos en este estudio son muy próximos a los reportados por Chen, W. et al. [23], que manifiestan haber obtenido un espesor de $30 \mu \mathrm{m}$ en un acero 304 nitrurado con sales. Usando la ecuación (1) la velocidad de difusión de $\mathrm{N}$ fue estimada en $2.2 \times 10^{-12} \mathrm{~cm}^{2} / \mathrm{s}$ para $550^{\circ} \mathrm{C}, 3.22 \times 10^{-12} \mathrm{~cm}^{2} / \mathrm{s}$ para $565^{\circ} \mathrm{C}$ y $4.63 \times 10^{-12}$ $\mathrm{cm}^{2} / \mathrm{s}$ para $580^{\circ} \mathrm{C}$. A temperaturas altas ocurre una sobresaturación de átomos de $\mathrm{N}$ que difunden a gran velocidad y que promueven la formación de una capa nitrurada de gran espesor [24].

\section{CONCLUSIÓN}

Los residuos de un polímero termoestable (melanina) fueron utilizados para endurecer superficialmente un acero inoxidable AISI: 304 mediante la difusión de nitrógeno (nitruración). A las condiciones de prueba de: $550^{\circ} \mathrm{C} / 4$ horas, $580^{\circ} \mathrm{C} / 4$ horas y $565^{\circ} \mathrm{C} / 3$ horas, se obtuvieron durezas superficiales de 30.17, 30.0 y 29.9 HRc respectivamente; con un incremento en la dureza de 66.9, 66.7 y $66.6 \%$ cuando se compara con la dureza de la muestra sin tratamiento y en su estado de suministro (10 HRc). El endurecimiento por nitruración se debe a la formación de nitruros de Fe del tipo $\gamma^{\prime}-\mathrm{Fe}_{4} \mathrm{~N}$ y $\varepsilon-\mathrm{Fe}_{2} \mathrm{~N}$ presentes en una capa de compuestos y en una zona de difusión. El espesor de estas capas depende de la temperatura y tiempo de nitruración, así como de la cinética de difusión de átomos de nitrógeno que difunden hacia el interior del acero. El uso de los residuos de melamina para el endurecimiento superficial del acero AISI: 304 es una alternativa técnica viable de bajo costo y un proceso que considera el reciclaje de desechos plásticos

\section{RECONOCIMIENTOS}

Los autores expresan su agradecimiento al Laboratorio de Microscopía y Transformación de Fases de la Universidad Nacional San Agustín de Arequipa, por su apoyo en la realización de la caracterización por microscopía óptica.

\section{REFERENCIAS}

1. Prochazka, J., Pokorny, Z., Dobrocky, D. 2020, Service Behavior of Nitride Layers for Military Applications, Coatings, 10(10), 2-14. DOI: https://doi.org/10.3390/coatings10100975

2. Seitz, M., Hoffman, J., Rieth, M., Margraf, P., Senn, R., Klimenkov, M., Lindau, R. 2017, Assessment of industrial nitriding process for fusion steel applications, Nuclear Materials and Energy, 13(2017), 90-98. DOI: http://dx.doi.org/10.1016/j.nme.2017.05.015

3. Monteiro, W.A., Lima-Pereira, S., and Vatauk, J. 2017, Nitriding Process Characterization of Cold Worked AISI 304 and 316 Austenitic Stainless Steels, Journal of Metallurgy, 2017, article ID 1052706, 1-8. DOI: https://doi.org/10.1155/2017/1052706

4. Deng, X., Ju, D. 2016, Prediction of Phase Composition and Nitrogen Concentration During the Nitriding Process in Low-Alloy Steel, Materials Research, 19(2), 353-359. DOI: http://dx.doi.org/10.1590/1980-5373-MR-2015-0137

Downloadable from: Revista Boliviana de Química http://www.bolivianchemistryjournal.org 192

Volumen 38 N5. Año 2021 http://www.scribd.com/bolivianjournalofchemistry 
5. Fraczek, T., Ogorek, M., Skuza, Z., Prusak, R. 2020, Mechanism of ion nitriding of 316L austenitic Steel by active screen method in a hydrogen-nitrogen atmosphere, The International Journal of Advanced Manufacturing Technology, 109, 1357-1368. DOI: https://doi.org/10.1007/s00170-020-05726-8

6. Tyan, Y.Ch., Yang, M.H., Jong, S.B., Wang, Ch.K., Shiea, J. 2009, Melamine contamination, Anal Bioanal Chem, 395, 729-735. DOI: 10.1007/s00216-009-3009-0

7. ASTM American Society for Testing and Materials, 2001, Standard Test Methods for Notched Bar Impact Testing of Metallic Materials, E 23-01, USA.

8. Wang, J., Lin, Y., Yan, J., Zeng, D., Huang, R., Hu, Z. 2012, Modification of AISI 304 Stainless Steel Surface by the Low Temperature Complex Salt Bath Nitriding at $430^{\circ}$ C, ISIJ International, 52(6), 1118-1123. DOI: https://doi.org/10.2355/isijinternational.52.1118

9. ASTM American Society for Testing and Materials, 2001, Standard Test Methods for Rockwell Superficial Hardness of Metallic Materials, E 18-00, USA.

10. Keddam, M., Thirie, T., Marcos, G., Czerwiec, T. 2017, Characterization of the Expanded Austenite developed on AISI 316LM Steel by Plasma Nitriding, J.Min. Metall. Sect. B-Metall., 53(1), 47-52. DOI: https://doi.org/10.2298/JMMB151115026K

11. ASTM American Society for Testing and Materials, 2001, Standard Guide for Preparation of Metallographic Specimens, E 3-01, USA.

12. Bobadilla, M., Tschiptschin, A. 2015, On the Nitrogen Diffusion in a Duplex Stainless Steel, Materials Research, 18(2), 390-394. DOI: http://dx.doi.org/10.1590/1516-1439.337714

13. Yan, F., Chen, B., Yang, Y., Xu, Y., Yan, M., and Zhang, Y. 2021, A Novel Decarburizing-Nitriding Treatment of Carburized/throughHardened Bearing Steel towards Enhanced Nitriding Kinetics and Microstructure Refinement, 11(112), 1-10. DOI: https://doi.org/10.3390/coatings11020112

14. Sakoshi, K., Kagaya, Ch., and Kagaya, E. 2006, Development of Solid Nitriding Using Thermosetting waste Plastic, Solid State Phenomena, 118, 121-130. DOI: https://doi.org/10.4028/www.scientific.net/SSP.118.121

15. Benlahreche, F.Z., and Nouice, E. 2017, Improvement of Surface Properties of Low Carbon Steel by Nitriding Treatment, Acta Physyca Polonica A, 131(1), 20-23. DOI: 10.12693/APhysPolA.131.20

16. Liu, X., Mao, Ch., Wu, M., Cai, E., Dai, M., Hu, J., 2020, Effect of Salt Bath Nitriding Time on the Performances of 304 Stainless Steel, Acta Metallurgica Slovaca, 26(1), 4-6. DOI: https://doi.org/10.36547/ams.26.1.458

17. Borgioli, F. 2020, From Austenitic Stainless Steel to Expanded Austenite-S Phase: Formation, Characteristics and Properties of an Elusive Metastable Phase, Metals, 10(2), 187. DOI: https://doi.org/10.3390/met10020187

18. Kondamuru, L., Bairi, S.Ch., Lakshmi, A., Subbiah, R. 2020, Assessment of Properties on AISI430 Ferritic Stainless Steel by Nitriding process, In E3S Web of Conferences, 184, 01020. DOI: https://doi.org/10.1051/e3sconf/202018401020

19. Marwan, N., Arbilei, N. 2018, Liquid Nitriding of Stainless Steel 316L to Improve Fatigue Properties for Orthopedic Screws, AlNahrain Journal for Engineering Sciences (NJES), 21(04), 508-515. DOI: http://doi.org/10.29194/NJES.21040508

20. Lin, Y., Wang, J., Zeng, D., Huang, R., Fan, H. 2013, Advance Complex Liquid Nitriding of Stainless Steel AISI 321 Surface at $430^{\circ} \mathrm{C}$, Journal of Materials Engineering and Performance, 22(9), 2567-2573. DOI: 10.1007/s11665-013-0545-8

21. Ferreira, L.M., Brunatto, S.F., Cardoso, R. 2015, Martensitic Stainless Steels Low-temperature Nitriding: Dependence of Substrate Composition, Materials Research, 18(3), 622-627. DOI: http://dx.doi.org/10.1590/1516-1439.015215

22. De Araujo, L., Goncalves, A., De Araujo, F., Ferreira, M.O., De Souza, R.M., Cavalcante, L.S., Nunes, L.C. 2020, Effect of plasma nitriding time on the structural and mechanical properties of AISI-O1 steel, Engineering Reports, 2(12), 1-9. DOI: https://doi.org/10.1002/eng2.12279

23. Chen, W., Xia, M., and Song, W. 2020, Study on the Anti-Friction Mechanism of Nitriding Surface Texture 304 Steel, Coatings, 10(6), 554. DOI: https://doi.org/10.3390/coatings10060554

24. Borgioli, F., Galvanetto, E., and Bacci, T. 2019, Surface Modification of Austenitic Stainless Steel by Means of Low Pressure GlowDischarge Treatments with Nitrogen, Coatings, 9(10), 604. DOI: https://doi.org/10.3390/coatings9100604

Downloadable from: Revista Boliviana de Química http://www.bolivianchemistryjournal.org
193

Volumen 38 N55. Año 2021

http://www.scribd.com/bolivianjournalofchemistry 